\title{
20. Improving Research Impact by Better Understanding Change: A case study of multidisciplinary synthesis
}

\author{
Gabriele Bammer
}

The requirement to demonstrate research impact is growing in prominence. It is no longer sufficient for research to provide greater understanding of a problem or issue. Researchers and those who fund them want to see findings used to bring about an improvement. Evaluation of research, and of research organisations, is therefore increasingly focused on measurement of outcomes in terms of policy change, improvement in professional practice, changes in community behaviours and attitudes, and patents and other indicators of commercialisation. This chapter examines research impact and the ability to improve it through the lens of better understanding change.

The focus of this chapter is on a key insight which has been largely neglected in considerations about research impact, but which is highlighted by the 18 disciplinary and practice perspectives on change in this book. This is that research findings enter a dynamic world, where everything is changing all the time. As researchers we sometimes operate as if the world is static, just waiting for our findings in order to decide where to head next. Instead, for research to have impact, researchers must negotiate a constantly changing environment.

The first part of this chapter draws together threads from the preceding chapters and the symposium discussion to describe the inevitability of change, as well as to examine continuity and conservation, inertia and resistance to change, and the propositions that change does not necessarily lead to improvement, that success is in the eye of the beholder, and that any attempt to influence change can have unpredictable outcomes.

The second part of this chapter examines three topics thrown into sharp relief when research impact is seen as involving a process of negotiating a dynamic change environment. It deals first with the challenges of managing opportunity costs, transaction costs and potential compromises to integrity. These require diverse levels and forms of research impact to be validated, which is the second topic considered. An additional requirement is a reward system that can encompass the complexity of research impact in a dynamic change environment. This is addressed in the third topic: a new publication culture as the primary assessment structure. 
Finally, an appendix to the chapter provides a brief description of multidisciplinary research and the synthesis of multidisciplinary perspectives, as well as of the emerging discipline which underpins this project, Integration and Implementation Sciences (I2S). As explained in the appendix, a multidisciplinary approach invites experts from different disciplines and practice areas to tackle the issue under consideration (in this case, change) as they see fit, which provides an array of rich perspectives. One or more syntheses are then tailor-made around topics of interest to the synthesisers (in this case there is one synthesis focused on research impact). As demonstrated in this chapter, not all of the perspectives are drawn on equally in the synthesis, and there are many valuable insights on change provided by the disciplinary and practice experts that are not used (because they are not directly relevant to the synthesis topic). A synthesis does not therefore absorb or replace the multidisciplinary perspectives; instead it draws on them selectively to illuminate a particular topic.

\section{The dynamic change environment}

Lindell Bromham's chapter on evolutionary biology is a potent reminder that we live in an environment of constant change and that everything is connected, as demonstrated by bringing together the following three quotations:

Evolutionary change is continuous and inevitable: nothing stands still in the biological world ...

The world around us is the product of evolution: not just the actual biological organisms such as the trees, birds and insects, but also many features of the environment such as the soil, atmosphere and buildings, which have been constructed by the actions of organisms over time. This life-built environment is in a constant state of change, as are all the biological lineages that inhabit it ...

No species exists in isolation ... Species both create and respond to changing environments, creating a tangled web of interactions in space and time.

At the human scale, Peter McDonald's chapter on demography also focused on the inevitability of change in that discipline's concentration on 'childbearing, death, disease, disability, migration, entry to and exit from relationships; education and employment; and housing types or tenures'. 
A similar point about the dynamics of change was made by Kate Carnell (personal communication, $2013^{1}$ ), reflecting on life as a politician:

What we are dealing with now isn't what we were dealing with five years ago or 10 years ago ... So change is inevitable. So politics is, I suppose, about attempting to ensure that your part of the world is anticipating that change, ahead of that change, and maximising the potential of that change. And also, why would you go into politics if you didn't want to change anything? It's not a great lifestyle. I mean you wouldn't do it for the lifestyle or for the money.

As Michael Wesley commented on Kate Carnell's chapter, 'It's a bit like the old John Howard line, reform is the ever receding finish line' (personal communication, 2013). ${ }^{2}$

The dynamics of change in business were described in Sarah Pearson's chapter, which highlighted competition as the driver of change, as in the following quotations:

Without the threat of competition most companies would not consider making the necessary changes for fear of having to deal with their associated challenges ...

Change is hard. It requires energy and a compelling reason to jump on board: energy from those leading change, and energy from the followers ...

The drive to remain competitive can provide the energy to help individuals and organisations accept new ways of working and delivering value.

Change is not, of course, uniform. Mark Stafford Smith pointed out the importance of 'the tempo and scale of change ... that's about a rate of change as opposed to a direction or degree of change' (personal communication, 2013). In terms of tempo, Lindell Bromham highlighted that 'no one, including Darwin, ever expected that the pace of morphological evolutionary change would be uniform over time' (personal communication, 2013). Grant Wardlaw's chapter on security-based intelligence adds further complexity by demonstrating that there

\footnotetext{
1 Quotations labelled 'personal communication, 2013' are taken from the transcript of the two-day symposium which brought together most of the chapter authors (see Chapter 1 for a description of the process). 2 John Howard was the Australian Prime Minister from 1996-2007. In 2005 he is reported to have said, 'Economic reform in this country, and the challenge of economic reform, is like participating in a race towards an ever receding finishing line' (The Age, 27 August 2005; www.theage.com.au/news/national/howardvows-to-stand-firm-over-labour-reforms/2005/08/27/1124563060427.html), and 'Economic reform is like participating in a race towards an ever-receding finishing line' (The Sydney Morning Herald, 15 October 2005; www.smh.com.au/news/national/the-real-deal/2005/10/14/1128796703087.html).
} 
can be major discontinuities, making the new situation 'almost unrecognisable'. Until fairly recently this field was characterised by slow-moving 'intelligence puzzles', now replaced by fast-moving 'intelligence mysteries-questions whose answers are inherently unknowable in detail (often because even the targets do not know precisely what they are going to do until they do it)'.

Scale of change can be illustrated by required responses. Some responses, for example, are constrained to an individual, or community, or a geographical region. Others, like global environmental change, have to be 'responded to at every level from households through to international UN [United Nations] level. So there's no way you can think about this problem without thinking about the multi-scale nature of it. And that's true in time as well as space, as well as organisational scale' (Mark Stafford Smith, personal communication, 2013). Beverley Raphael (personal communication, 2013) added another dimension, contrasting 'change that we can impact on' with 'change which is out of control', and likening the latter to a tsunami.

Finally, embracing the inevitability of change is not universal, as Francesca Merlan (personal communication, 2013 $3^{3}$ ) noted in her comments on Lindell Bromham's chapter: 'One of the useful things in this account for the social sciences is the easy acceptance of the inevitability of change. It's going to happen and ... [is] inherent in the very process of reproduction itself ... This may ... help social scientists feel easy about the same thing.'

The rest of this section describes different dimensions of the dynamic change environment:

- continuity and conservation-i.e. stopping change from happeningrequire work

- there is often inbuilt inertia or resistance to change, so that once something exists it can be hard to get rid of

- even though change happens all the time, it does not necessarily lead to improvement

- success is in the eye of the beholder

- any attempt to influence change can have unpredictable outcomes.

3 Francesca Merlan was unable to attend the symposium, but provided written comments which are used here and elsewhere in this chapter. 


\section{Continuity and conservation require work}

A corollary to the inevitability of change is that stopping change from happening requires effort, whether this is conservation of artefacts or environments, or continuity in social affairs and behaviour.

Ian MacLeod's chapter on materials conservation illustrates the inevitability of change in the form of decay and provides some insight into the range of techniques available to prevent and inhibit decay:

For every type of material there is a specialist conservator who knows how to stabilise and preserve elements of our material culture that range from ephemeral art works to plastic furniture and toys to digital media.

For example,

Willing bands of seamstresses ... worked on textiles. Faithful sacristans have worked in churches for hundreds of years conserving the textiles that form the basis of ecclesiastical garment collections. They used traditional methods of stitching down degraded fibres of sacred and preciously embroidered fabrics onto sympathetic new support structures.

Nevertheless, as he pointed out in the symposium discussion, 'There gets to a point of diminishing returns, and you've got to make the hard decision of what do you preserve and what do you let go, and let decay take its natural course' (Ian MacLeod, personal communication, 2013).

When it comes to environments, Lindell Bromham (personal communication, 2013) pointed out that 'we automatically think of conservation as, 'put a fence around it, keep it as it is'. That's not possible. You can't do it.'

Conservation is similarly challenging in social affairs, as Francesca Merlan described in her chapter on anthropology: 'Both change and continuity involve expenditures of energy; both are actively produced in the human world, and discerned in particular circumstances.'

Lindell Bromham (personal communication, 2013), reflecting on Francesca Merlan's chapter, embellished this point:

Genetic change is inevitable, mutations are always arising; if you see sequences staying the same in the genome, it's not because nothing's happening, it's because something very active is happening, which is the removal of any changes that arise. So you could think of the same thing perhaps in ritual, that if you see it staying the same, it's not because it's failed to move, but because people have actively kept it the same. 
We see therefore that keeping artefacts, environments and social practices the same requires energy. There are also additional dimensions in the social realm, where power and legitimacy can be connected to continuity.

In highlighting insights provided by the discipline of sociology in his chapter, Craig Browne pointed out:

It is probably a banal, though nevertheless true, insight that individuals towards the top of a field or social hierarchy generally argue for either the preservation of the existing order or for managed change.

Furthermore he alerts us to the fact that:

Sociologists often think about change in a comparative and constructionist manner, since they seek to demonstrate that what is assumed to be natural and permanent is actually a product of historical processes and culturally specific practices.

He drew on theorist Pierre Bourdieu to elucidate the disguising of the effort that goes into maintaining continuity:

The denial or veiling of effort is part of the logic of reproduction, because social legitimacy is regularly achieved by the perception of a person and practice as given, natural and normal.

Francesca Merlan used her work with Indigenous Australians to highlight political pressure to demonstrate continuity in order to achieve land and other rights:

There are now often aspects of reparations politics - in the case of indigenous peoples, for instance, land and native title or other similar claims - that ask of them to demonstrate their continuing attachment to lands and traditions as the requirement and justification of those claims. This supports and amplifies concerns for authenticity, traditionality and unchangingness of law and custom among those people which may constitute for them a source of pride, as well as a considerable problem to the extent that they no longer are as they were, and do not live up to such expectations.

In her chapter she went on to argue that these expectations are unrealistic and that anthropologists have not gone far enough in exploring this challenge. 


\section{There is often inbuilt inertia or resistance to change, so that once something exists it can be hard to get rid of}

The effort that goes into producing continuity can also lead to structural inertia or resistance to directed change. Michael Wesley's chapter on international relations shines a powerful light on this topic. He argued that contemporary international relations 'tends towards routines and stasis, accreting over time structures and forces of inertia that are periodically overwhelmed by underlying change'. Inertia results from 'the increasing routinisation of international life', where international institutions, for example,

preserve the power structures and expectations that existed at the time of their establishment. This makes them extremely hard to adapt to the evolution of international relations, and equally hard to abolish, given the reputation capital and hopes that have been invested in them over the years. The solution to the declining relevance of existing institutions is rather to simply establish new ones - which turn out to be equally inflexible and of declining relevance over time.

He drew on other work to add:

Inertia is the result of the difficulty and cost of change and the investment of the powerful in current ways of doing things. Several factors play interlocking roles in supporting inertia and routinisation. Institutions and relationships often embody high start-up and sunk costs. Often they were negotiated at an opportune time that is unlikely to recur; inevitably they are seen, for all of their defects, as being better than no institution, agreement or relationship at all. Existing institutions exert strong learning effects, by favouring those who adapt their strategies and expectations to the existing rules. These are buttressed by adaptive expectations that favour existing ways of doing things over innovation. Bureaucratic objectives tend to be moulded by institutional possibilities, rather than vice versa. Routinisation incentives tend to multiply activities that comply with existing structures and crowd out opportunities for innovation. Competency traps breed familiarity with existing rules and bureaucratic training becomes oriented to most effectively using those rules.

In his chapter Grant Wardlaw drew on the work of others to highlight an additional cause of inertia, namely that at least some agencies 'are built to be reliable, consistent and predictable - not adaptive'. He argues, 'the intelligence system as a whole is not designed to cope well with change ... Particular parts of the system have, in effect, been designed not to adapt.' 
Inertia highlights the point that while change is inevitable, the speed of change is far from uniform and may be glacial in some areas. As Craig Browne pointed out in his chapter, inertia is also a prime concern in sociology:

Sociological research has regularly demonstrated that change has been limited in major areas of social relations, especially those to do with longstanding inequalities. Sociologists quite often find discrepancies between the widespread social perceptions of changes in the circumstances of subordinated social groups and their actual conditions. For example, the overall remuneration of female labour compared to male labour has not changed as much as might be presumed from the enacting of equal pay legislation in Australia several decades ago. These kinds of discrepancies highlight the complexity of explaining change and the constraints upon changing enduring dimensions of society.

Drawing again on Pierre Bourdieu, he added another explanation for inertia:

Many embodied social practices may be quite resistant to conscious modification, yet represent the unrecognised components of social assessment ... Social actors have an intuitive understanding of probability in social life. Consequently, they are always making implicit assessments about what they can achieve and the amount of effort that would be involved in significant change.

In her chapter, Kate Carnell described how the system in which politics is embedded leads to inertia and resistance to change, as well as the frustration that this causes:

Politicians want to make their electorate, state and country a better place. Most start wide-eyed and optimistic. The mechanisms of government and the sheer difficulty of bringing about change often produce disenchantment and cynicism. The impediments to delivering real change start with your own party and supporters. Then there is the media, our relatively short political cycle, the bureaucracy, and intolerance to any political leader's policy failure.

As she pointed out in her chapter, in addition to inertia, change fatigue is a potent factor:

With new policy and change come some great successes, some average outcomes and a few failures. And the failures are seized on by both the opposition and the media. The media say that good news does not sell, so a feeding frenzy over a government initiative that has not worked is great copy ... The longer politicians are in power, the more things there will be that have not gone quite right ... Change fatigue is a real 
thing in politics. With change comes risk and with risk comes the very real possibility of failure (or at least lack of success). I believe this is the reason many governments become increasingly risk-averse the longer they spend in office.

Finally, Sarah Pearson (personal communication, 2013) described how inertia is also a problem in business, and gave one example of a mechanism which businesses are developing to prevent inertia from setting in:

It's the same in innovation. It's actually very easy to start something, or relatively easy to start something new, a change or something new, actually stopping something, the change that stops something is really, really hard and in innovation you call it fast failure. So companies are really trying to learn how to fail fast ... they want to know when they know they've failed.

This is achieved using 'stage gate processes. So you have your projects managed and they have specific timelines where they have to meet certain criteria and if they don't-dead.'

\section{Change does not necessarily lead to improvement}

Evolutionary biology is again very helpful here, demonstrating that much change is self-generating and often negative and maladaptive. Lindell Bromham (personal communication, 2013) pointed out:

We often think of both evolution and other kinds of change, for example institutional changes, being driven by a need to improve but actually there [are] an awful lot of trends in change that are just driven by underlying generation of change and may not actually be positive.

The point that change does not necessarily lead to improvement is also fundamental to Mark Stafford Smith's chapter on global environmental change, where he cited ideas about nine planetary boundaries and the necessity to keep change within a 'safe operating space ... to have confidence that the planet will continue to function in the relatively benign state that it has during the past 8000 years. In this time, modern civilisation, agriculture, cities and technologies have developed.' (There is evidence that three of the planetary boundaries have already been transgressed.) 
Debate about change and improvement is highlighted in Michael Wesley's chapter which describes three competing views about the direction of change, one which sees change as improvement and two which do not. They are:

- teleology, which sees change as 'progressive and unidirectional' to a future that is 'safer, more prosperous, more just, and more sustainable'

- cyclicality, which argues that 'periods of peace and prosperity ... usually come to an end in a catastrophic conflict that resets the basic ordering of power in the world'

- episodism, which views change as 'episodic and unpredictable in its timing, extent, and direction'.

Sociology has also grappled with this issue, specifically in terms of change and progress, as Craig Browne noted in his chapter. In particular, 'sociologists are now more reluctant than their predecessors to equate change with progress'. This is particularly evident in interpretations of modernity:

It had previously been presumed that modernisation would lead all societies or nation states to share a common pattern of institutions and cultural values. In effect, change would lead to a convergence in the form of modern societies and underpinning this assumption was the equating of modernising change with other notions, especially those of progress, rationalisation and development.

But as he also pointed out,

Assumptions about the overall development of society and social evolution are probably still implicit in sociological analyses that are addressed to other topics, like gender and education, sexuality and consumption.

The point here is that any underlying assumption that change equates to improvement or progress should be questioned, rather than assumed to be correct. Other considerations about the direction of change-perceptions of success and unpredictability - are dealt with in the following sections.

\section{Success is in the eye of the beholder}

Change is 'not value neutral. It depends on who you ask' (Simon Chapman, personal communication, 2013). The point was illustrated by the following exchange at the symposium. Michael Wesley (personal communication, 2013) to Kate Carnell: 'I think that quite often change is in the eye of the beholder, and it made me start to think that someone who disagreed with you politically might look at your period as Chief Minister and say, "Well they did nothing, they were 
a do-nothing government"'. Kate Carnell (personal communication, 2013): 'I agree with that. I think that probably the people who perceive that about my time would say not that I didn't do anything but I did a lot of bad stuff.'

At the symposium Mark Stafford Smith (personal communication, 2013) raised the issue of: 'whether you can predict whether it's going to be good or bad which is a different challenge'. Craig Browne (personal communication, 2013) added:

The problem isn't so much knowing that retrospectively, we can know retrospectively what is good or bad change ... I think the difficulty is more practical in a sense. Should we initiate change on the assumption that it will lead to a good? ... How we decide on that of course is a contested issue.

While this issue was not explored in more detail, it is significant for research impact.

\section{Any attempt to influence change can have unpredictable outcomes}

The inevitability of change, the interconnectedness of what is changed and the various aspects of change dynamics discussed above mean that attempts to influence change can have outcomes which are unpredictable. As Jim Butler (personal communication, 2013) noted, 'by the very fact that we're talking about change, we're going to talk about uncertainty'. Even when, as Lindell Bromham described in her chapter, the mechanism for change is 'breathtakingly simple', as for evolution, the outcome can be 'devilishly complex'. The reason is that 'species both create and respond to changing environments, creating a tangled web of interactions in space and time'. This interconnectedness and ever-changing environment and context hold true for the social aspects of life as well.

One aspect of this is 'the arms races between predators and prey, plants and herbivores, pathogens and hosts' described by Lindell Bromham in her chapter. This has a direct parallel in intelligence, as described by Grant Wardlaw in his chapter: 'Adaptation is a two-way street. As intelligence gets better at finding and understanding its targets, the smarter targets also adapt to evade intelligence.' 
Unintended consequences are a key dimension of unpredictability. Craig Browne drew on the work of Michel Foucault to demonstrate that outcomes can be the opposite of those intended:

The notion of unintended consequences is a feature of many sociological conceptions of change, but Foucault's historical genealogies show how movements for humane punishment, sexual liberation and liberties were themselves implicated in the extension of power and resulted in the more intensive disciplining of prisoners, the regulation of sexual identities, and the consolidation of governmentality.

Unintended consequences can also feature even in relatively straight-forward circumstances, such as materials conservation, as described in Ian MacLeod's chapter. For example,

Months or years after an apparently successful intervention, the surface of ceramics can become covered with white effloresences of hydrated calcium acetates as the low-fired earthenware corrodes in an atmosphere containing acetic acid, coming from the decay of old wooden showcases. These storage cabinets were often made of oak, which is one of the worst timbers to use, but it is one that had been traditionally used in institutions in Europe.

While these are easier to manage than major unexpected social changes, unintended consequences at all scales can be important for research impact.

Another dimension of unpredictability is unexpected events. Peter McDonald demonstrated the challenges of prediction, citing two demographic projections for Australia's future population in his chapter which 'do not even overlap with each other, despite just a three-year difference in the publication of their results'. As he explained, 'These two projections differed dramatically from each other because, between the two projections, Australia's international migration level rose very considerably.'

In discussing demographic predictions at the symposium, Lindell Bromham (personal communication, 2013) added:

Of course there will always be completely unexpected changes ... Consider the HIV. No one could have predicted HIV bursting onto the world stage in the late 70s and early 80s and yet HIV has reversed life expectancy trends in Africa. So anyone who was projecting forward on life expectancies in Africa in the 1970s got it completely wrong because they are going backwards now. 
Another aspect is knowing that change is required, but not knowing if it will happen, as Mark Stafford Smith pointed out in his chapter for global environmental change:

It is certain that there is major change occurring on a decadal to centennial timeframe, but there is considerable uncertainty about where that change will end up and what impacts it will have. A large part of this uncertainty is a result of not knowing whether humanity will choose to respond to mitigate the changes.

Overall, there was little consideration of the importance of context as a factor underpinning unpredictability, except in how it limits what is possible. This was highlighted in the quotation from Karl Marx (1977, 301; first published 1852) cited by Craig Browne in his chapter:

'Men make their own history, but they do not make it just as they please; they do not make it under circumstances chosen by themselves, but under circumstances directly encountered, given and transmitted from the past.'

Mark Stafford Smith (personal communication, 2013) made a very similar point in referring to 'path contingency', where decisions made today limit options available in future.

There are other aspects of unpredictability that were not covered or teased out in the chapters or the symposium discussion:

- change depends on interactions between people, who have their own values and interests and may therefore behave in unexpected ways

- serendipity can be a key factor

- impact may not be in proportion to the quality, veracity or importance of the data

- decisions often have to be made without a solid evidence base.

All of these are important in considering research impact. 


\section{Research impact as a process of negotiating a dynamic change environment: Issues and consequences}

Laying out the complexities of the dynamic change environment highlights the challenges of achieving and assessing research impact. Researchers seeking to influence change are buffeted by a range of forces - some supportive, some hostile, some neutral - and even in the best circumstances unpredictable outcomes may occur. There is no sure way to negotiate a path through those forces and there are no guarantees of success. No consequences at all or adverse unintended consequences are always real possibilities. Those assessing research impact must be sensitive to the realities of the vortex of change-for example, that hard work and skill are not always rewarded, that luck may play a large hand, and that good intentions may be punished with bad outcomes.

Focusing on change and its complexities highlights three topics relevant to research impact which warrant particular consideration. The first is the challenges that must be recognised and managed - opportunity costs, transaction costs and potential compromises to integrity. These in turn demonstrate that there can be no one size fits all for researchers seeking to achieve impact. The second topic therefore explores the requirement for diverse levels and forms of research impact to be validated and presents three frameworks which provide a systematic way to document the diversity. Finally, the process of rewarding research impact must be able to encompass this diversity, as well as the complexities of achieving change. This is addressed in the third topic, which proposes a new publication culture as the primary assessment structure. While these issues were not addressed specifically in the chapters or symposium discussion, both provide a range of illustrations for the points made.

\section{Costs and compromises: Three challenges to recognise and manage}

As research impact moves from being a relatively minor add-on to the main business of discovery and becomes a more significant aspect of the work of researchers, the vagaries and difficulties of achieving research impact are thrown into greater prominence. These lead to three particular challenges - at both individual and institutional levels - which are teased out here. The first is opportunity costs, especially the trade-off between achieving new insights or discoveries and achieving impact. The second is grappling with the negative 
sides of achieving research impact, what may be called the transaction costs. Third is reputational risk; the challenges to integrity that can result from engagements and actions to achieve research impact.

\section{Opportunity costs}

Opportunity costs for individual researchers are centred on three limited resources: time, energy and expertise. Researchers need to allocate how much time they will spend on improving understanding and how much on achieving impact. Each requires not just hours, but also commitment, which can be thought of as the level of energy devoted to them. Finally the expertise required for achieving research impact is quite different from that required for improved understanding (discovery). And expertise not only has to be acquired, but also maintained, by staying on top of new and improved methods and processes as they are developed.

A simple example is the academic papers which researchers are trained to write. Writing to reach the media, policymakers, business or civil society actors requires different styles from that of the academic world. The necessary skills have to be learnt, which demands time and energy. With social media providing new outlets, researchers also have to stay up to date, assessing when and what social media outlets may have the best reach into the audience of interest, and learning how to use them.

Furthermore, written evidence is not necessarily the most effective form of communication to these groups. At least sometimes, images and prototypes may be more useful. Dee Madigan and John Reid both highlighted the power of images in their chapters, with John Reid stating: 'Visual aesthetic imagery informed by science brings a human, high-fidelity, emotional dimension to science communication'. Influencing change in commercial products is likely to be most successful when there is a prototype, as Sarah Pearson (personal communication, 2013) described:

To get the company [Cadbury] to change I had to show them something tangible. I started with pictures: didn't work. I started with something they could just touch: didn't work. They had to be able to eat it. So I think when you are trying to bring about change some tangible way of explaining what the change is about really helps.

Again, developing effective images and prototypes requires time, energy and skill. 
Further illustration of opportunity costs, along with the broad range of skills required, is provided by the intense engagements with change described by Simon Chapman. He provided insights into two ways in which researchers contributed to tobacco control efforts: by helping prepare the ground and by helping ensure success.

Let us start with helping prepare the ground. Reflecting on an interview he undertook with former Australian federal government health minister, the Hon. Nicola Roxon, about her decision to introduce plain packaging legislation for tobacco products, Simon Chapman (personal communication, 2013) said:

The point that she made repeatedly is that it doesn't just happen. You don't sit bolt upright in bed one night and go: 'Someone has given me an idea-let's do it'. And she talked about the notion that there was a confluence of big shoulders to stand on that had come through historically, which had conditioned both her colleagues and the community that this was something which would be a no-brainer and they could do.

One way researchers can help prepare the ground is by developing a high, credible public profile through publicising their research and its relevance to the issues. Again Simon Chapman from his chapter:

I 'meet' these people [politicians and senior policymakers] many times each month, often at times when they are most relaxed and receptive. I had never met Nicola Roxon until invited to her 2008 prevention forum. As we met, she said 'I feel I have known you most of my life', referring to my long involvement in news media. So while I had never previously had the opportunity to put proposals to her directly, it was immediately clear that I had done so on innumerable occasions as she awoke to radio news and commentary, in her car as she drove to work or around on weekends, via her newspapers, through television news and via any online feeds she may have followed. Her cabinet colleagues would also all need to be voracious news consumers, so when health portfolio items arose, they too would be acquainted with the public narratives about these issues that have been circulating in news media.

Simon Chapman (personal communication, 2013) enlarged on the need for long-term involvement:

Debates obviously evolve. The start of a debate is seldom where a debate ends up, and I think those of us who are involved in pushing for social change recognise the natural history of a debate and that there are times when you go out and advocate for change and you know that you are going to be treated like a lunatic, but that if you are just a bit 
patient, in ... five to 10 years ... you start seeing conservatives alluding to the same things that you were arguing for as a radical and it becomes mainstream and before you know it it's legislation.

In terms of helping ensure success, in his chapter Simon Chapman described what happened when the introduction of plain packaging was announced: 'From that moment, those working in population-focused tobacco control in Australia did little else for the next two years than concentrate our efforts to ensure the announced bill would be passed.' This included helping 1) develop new effective packaging, 2) meet national and international legal challenges, 3) draft legislation, and 4) get cross-party political support.

It is clear that such activities require time and energy as well as skills in communication, including effective framing of issues and lobbying. These in turn require an understanding of the targets for these activities. Simon Chapman was seeking to influence a section of government, but for other research the potential end-users may be in business or civil society. Understanding the end-users is a time-consuming task and can be daunting. For example, it is a substantial undertaking to identify the relevant departments, committees and individuals (in government, for instance), as well as existing policies and likely positions that will affect how the research findings are received, and then to figure out when and how best to provide the research evidence and what follow-up is desirable, especially to achieve action and to counter opposition.

In addition to understanding where and how to target their efforts, researchers also need to appreciate the context which guides what is possible at that particular time. These were described in different ways by Craig Browne and Christine Nixon. In his chapter, Craig Browne pointed out that 'major changes in social structures are conditioned by the strains, conflicts and contradictions of a social order'. Christine Nixon made this more concrete in her chapter, highlighting the need to deal with 'the home of real power structures, the history of successes and failures, the "this is the way we do things around here" approach, the beliefs and values, the fears and unwritten control mechanisms'. Understanding and negotiating these can be critical in achieving change, including research impact. A particular relevant skill is recognising and using leverage. In Christine Nixon's case these were events and opportunities 'that helped or required us to focus'. She described, for example, how she used problems within the drug squad to focus on her goal of corruption prevention and concern about large numbers of underworld murders to strengthen criminal investigation.

Seeking to achieve research impact therefore requires time, energy and expertise, with a trade-off for individual researchers between conducting research to improve understanding and seeking to achieve change based on 
their research findings. Individual researchers will differ in how they prefer to make this trade-off and, as I argue in a subsequent section, such diversity must be accommodated.

Let us now move to opportunity costs at the institutional level, where the primary limited resources are funding and reputation. Only funding will be dealt with here. Universities and other research organisations have to decide what proportion of resources to dedicate to supporting research focused on discovery and how much to supporting impact.

In research organisations, supporting impact commonly occurs through the media offices, offices for commercialisation and academic centres on science communication. While such facilities provide important assistance to researchers seeking to achieve research impact, they cover only a small part of the terrain. Researchers who want to influence the government policy process, for example, are often left to their own devices in figuring out how the process works, who to target, how best to engage, and how to navigate the circumstances of the time, as outlined earlier. There is little opportunity for them to systematically learn from others or to contribute the knowledge and expertise they gain to others. This leads to considerable and wasteful reinventing of the wheel.

In my book Disciplining interdisciplinarity (Bammer, 2013), I argue for the establishment of a new discipline (Integration and Implementation Sciences or I2S), as a repository for the expert knowledge required to strive for research impact. ${ }^{4}$ Academics trained in I2S would specialise in the implementation process and be involved as collaborators on projects that aim to have research impact (just as statisticians are included on projects that involve quantitative analysis). These specialists would bring to the partnership the best current understandings of how to achieve impact, including effective ways of framing issues, identifying targets, and interacting with them. Establishing such a discipline provides a mechanism for capturing a range of pertinent experience and for building a body of relevant theory and practice. I2S provides an answer to the opportunity cost dilemma for individuals by focusing on effective collaborations. Just as individual researchers generally do not have all the skills for improving understanding in their area of expertise and overcome this through team work, they could also find collaborators for some or all aspects of research impact by partnering with I2S specialists.

4 I argue that research integration is also essential for effective implementation or impact. This is particularly relevant for teams tackling complex problems. In these case I2S specialists would contribute skills in, for example, effective ways of combining different disciplinary insights, bringing to bear relevant stakeholder perspectives, dealing with the system as a whole and minimising adverse unintended consequences. 
While this could solve the opportunity cost for individuals, it highlights the opportunity cost at the institutional level. Establishing such a discipline requires funding, which has to come from somewhere. And leaving the establishment of a new discipline to one side, institutions face the same issues if they try to improve support by expanding their current activities in media liaison, commercialisation or science communication. Indeed individual researchers often ignore this institutional trade-off when they lobby for more support.

Managing opportunity costs on either the individual or institutional level is not straightforward, and there are no easy answers. But if effective research impact is to become a norm, the issues must be brought into the light and sorted out.

\section{Transaction costs}

This section explores costs incurred when researchers seek to have impact, picking up on the comment made by Christine Nixon in her chapter: 'You might be in for a rough ride'. There is often resistance from those with interests in maintaining the status quo, who can use a range of tactics. Peter McDonald (personal communication, 2013) described one such tactic: 'If you are sticking your head up and you are sticking your head up often ... you quite often get labelled ... It's a kind of way to prevent change'.

Criticism can also come from academic colleagues, as Simon Chapman described in his chapter. Some researchers he interviewed

had encountered colleagues who questioned the motivation of mediaengaged researchers, seeing their promotion of research as an unseemly, 'ego-driven, empire-building' activity. These critical colleagues dismissed those who engaged with the media as 'self-promoting' or 'show ponies', terms 'designed to circulate the view that proper science is science which does not seek to promote or publicize its findings'.

Attacks can also be extreme, although these were not discussed in the chapters or at the symposium. For example, when a recommendation to trial heroin prescription based on research that I led achieved government support (subsequently withdrawn), the editor of a prominent newspaper accused me of being a drug dealer. Similarly, government uptake of Bruce Chapman's research on an income-contingent loans scheme to fund higher education (described further below) led to highly personal attacks, especially from university students. These included a 'wanted' poster, accusing him of 'crimes against education' (Chapman, personal communication, 2009). 
By and large, researchers are poorly prepared for these kinds of attacks, especially in coping with the stress they can cause or in knowing how to effectively respond. Research institutions can also come under attack, which can range from pressure to sack the academics involved, to threats, or actual cuts, to funding.

There is little documentation or analysis of either individual or institutional attacks. There are advantages in closing down such attacks as soon as possible, by denying them the 'oxygen' of discussion. But the downside is that individuals and institutional leaders are largely on their own when such attacks occur and cannot easily draw on the wealth of previous experience - successful and unsuccessful - in handling attacks. As with managing opportunity costs, managing transaction costs is not straightforward. But again the issues must be brought into the open, so that they can be carefully considered and acted on.

\section{Potential compromises to integrity}

Integrity is the cornerstone of the research enterprise. Attacks on individuals and institutions discussed above are almost always based on impugning their integrity. Often the parties involved have acted with the utmost propriety, but not always. Indeed in seeking to achieve change there can be temptations to let 'the ends justify the means' that can destroy the trustworthiness of both individuals and institutions. This is probably the biggest challenge that needs to be addressed in relation to achieving research impact. Here I am not going to reflect on deliberate misconduct, but I am going to briefly discuss two ways of 'crossing the line' to illustrate the kinds of issues that need to be considered and dealt with.

The first is errors of judgment. Let us take an example from Kate Carnell's chapter on politics, where she described her regret at compromising her values to get her government's budget passed:

At one stage in my time as ACT Chief Minister, two of the three independent members whose support I needed to pass the government's budget refused to pass it unless I agreed to amendments to abortion laws ... I finally agreed to their demands but have regretted it ever since ... I was publicly committed to pro-choice, so what was I doing supporting these changes to the abortion laws? I should not have compromised on the issue.

Putting oneself in Kate Carnell's shoes, it is easy to understand how an unwillingness to lose power (the consequence of the budget not being passed) combined with fatigue can lead to an error of judgment. Indeed we all make errors of judgment, often without as good an excuse as intense pressure or fatigue. This is pertinent to the issue of research impact, because errors of judgment are 
more visible (and fair game for opponents) when there is public engagement. Researchers and research institutions need to consider how errors of judgment will be handled.

A second way of crossing the line is losing sight of, and respect for, other people's values. There are many complex threads in this argument. I present one here as an illustration. While it is now commonly accepted that researchers, like everyone else, are influenced by their values, it can be argued that there is still a fundamental role for researchers to respectfully take other values into account in their work. This may often be incompatible with seeking to achieve impact. Take for example, the following insight from Simon Chapman's chapter:

It has long been banal to note that policy adoption is not simply a matter of presenting the best facts and evidence to policymakers and sitting back to watch evidence triumph over other considerations. With rare exceptions, policy entrepreneurs and advocates need to engage in serious, extended and highly strategic efforts to ensure that evidence is communicated in ways that make it publicly and politically compelling, so that inaction is not an option. Policy solutions need to be framed in ways that make their rejection problematic.'

Effective framing may involve ignoring and even denigrating other values. It is easy to get caught up in such processes. This leads on to a range of other issues, including when it is appropriate, and indeed essential, for researchers to take sides.

There is a potent interplay between transaction costs, potential compromises to integrity and the realities of political engagement, where even when researchers act with integrity, there is no guarantee that opponents will 'play fair'. These issues stem directly from understanding the complexities of achieving change which must be grappled with if research impact is to be more effectively achieved. Again there are no easy answers here, but the issues need to be recognised, discussed and managed.

\section{Recognising diverse levels and forms of research impact}

As outlined above, negotiating a dynamic change environment requires time, energy and different skills from those needed to undertake excellent research. There may also be unpleasant interactions with opponents, threats to funding and reputational traps. Add to this the vagaries of the process outlined earlier, including the unpredictability of outcomes and the subjectivity of success. Not surprisingly, while some researchers revel in the hurly-burly of a highly political engagement to drive change, for others this is anathema. It seems 
unreasonable, therefore, to require all researchers to conform to particular ways of achieving impact, and more sensible to validate a range of possibilities. The challenge then becomes how to document and assess numerous options in ways that acknowledge different inputs of time, energy, expertise and what might be called political capital.

As a starting point, three frameworks are presented to tease out key aspects of research impact and to illuminate its diverse levels and forms. They address:

- how research can have impact

- kinds of change

- where the research is targeted.

The frameworks were developed by combining insights from the preceding chapters and the symposium discussion with my previous work (Bammer, 2013). The aim is to give researchers and those assessing research impact a systematic way to describe what is being attempted and what has been achieved. An agreed systematic description is essential for effective evaluation, which is described in the next section. If the systematic approach looks useful, the next step will be to flesh out the frameworks, for instance, by testing them against a number of case examples.

\section{How research can have impact}

Research can have impact by informing, triggering or driving change (Figure 1). These require different skills and practices and also differ in terms of opportunity costs, transaction costs and potential challenges to integrity.

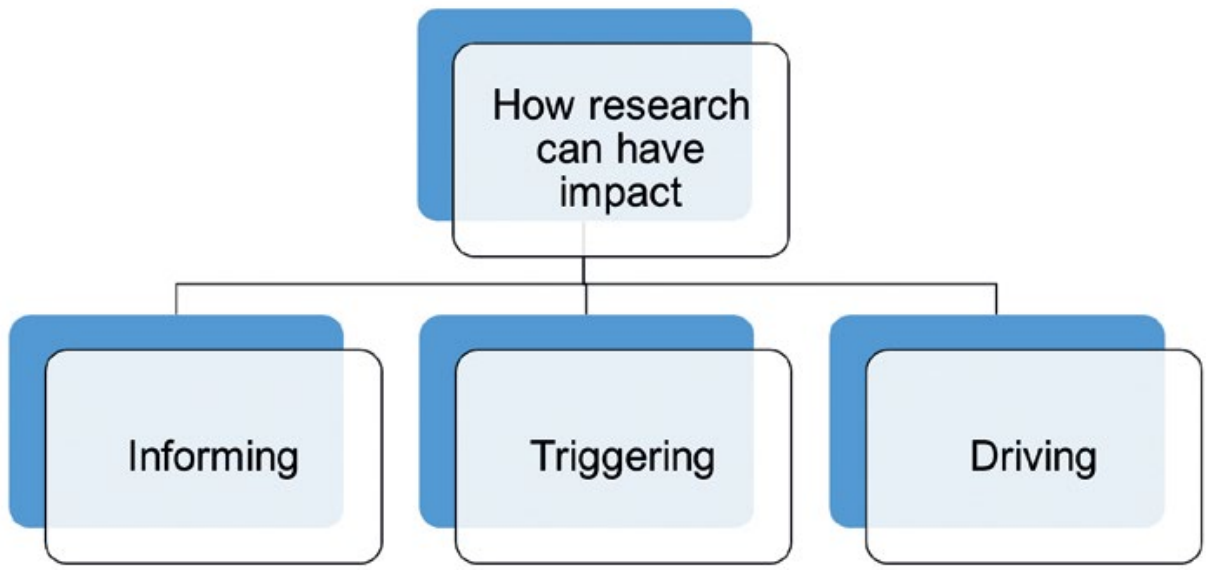

Figure $1 \mathrm{~A}$ framework for understanding and assessing how research can have impact.

Source: Author's illustration. 
Informing change involves providing all or part of the evidence base for change, including illuminating options for change. To use a piece of the quotation from Simon Chapman presented earlier, this is largely 'a matter of presenting the best facts and evidence' and is probably the most common way in which researchers seek to achieve impact, although there is significant diversity in the sophistication of the framing used. An example is Robyn Gillies' chapter which makes a case for altering Australian government policy using, among other things, a review of evidence from overseas experience.

Triggering change occurs when the research provides a catalyst for change. Common forms of triggering are producing a breakthrough finding or idea, and agenda setting. A breakthrough finding is often the solution to an essential missing piece of a research puzzle that allows a body of evidence to be employed in real-world change. The work of John O'Sullivan and colleagues which contributed to the development of wi-fi is an example. They discovered the critical technology that allowed information to be sent over many different frequencies and recombined at the receiver. ${ }^{5}$ A breakthrough idea generally provides an effective way forward on a recognised policy or practice issue. An illustration is Bruce Chapman's work on a loans scheme to fund higher education, where the breakthrough idea was that repayments would be contingent on income. ${ }^{6}$ Agenda-setting usually involves concerted action to promote a neglected issue to bring it to the attention of those responsible for making change happen. The reports of the Intergovernmental Panel on Climate Change ${ }^{7}$ provide an example.

Driving change results from investigation usually combined with direct liaison with those responsible for change, or actively concerned about it, including policymakers, business entrepreneurs or activists. The researchers may take an activist role themselves to promote implementation of their research findings. Often, research not only continues to accumulate evidence related to the change, but also evaluates changes which take place, investigates how to counter opposition and studies how to accommodate evolving circumstances. For example, research producing a new product such as a pharmaceutical or a machine is in itself generally not enough for commercial implementation. This may require ongoing negotiations with entrepreneurs or companies to demonstrate the potential of the product, overcoming vested interests who stand to lose market share if the product is introduced, and investigating tweaks to the product as problems emerge. Simon Chapman's chapter also provides an example of driving change, illustrating the research, interactions and actions involved

5 en.wikipedia.org/wiki/John_O\%27Sullivan_\%28engineer\%29.

6 The original idea and other potential applications are briefly described in Bruce Chapman (2014) 'Financing the future', in the online magazine Advance: crawford.anu.edu.au/files/uploads/crawford01_cap_anu_edu_ au/2014-12/summer_2014_advance_for_web.pdf.

7 www.ipcc.ch/. 
in the introduction of plain packaging of tobacco products. That chapter also provides insight into how this activity fits into a longer term research and activism campaign for tobacco control. Driving change is most likely to have significant opportunity and transaction costs, as well as reputational risks.

\section{Kinds of change}

Different kinds of change may also have different costs and risks, as well as requiring different skills and techniques for achieving them. This framework aims to tease out the main categories of change relevant for thinking about research impact, which may be useful both in describing research impact and also in assessing it. Let us start by categorising two primary types of impact:

- making change happen

- responding to particular proposed or ongoing change.

Within each of these there are subcategories (see Figure 2).

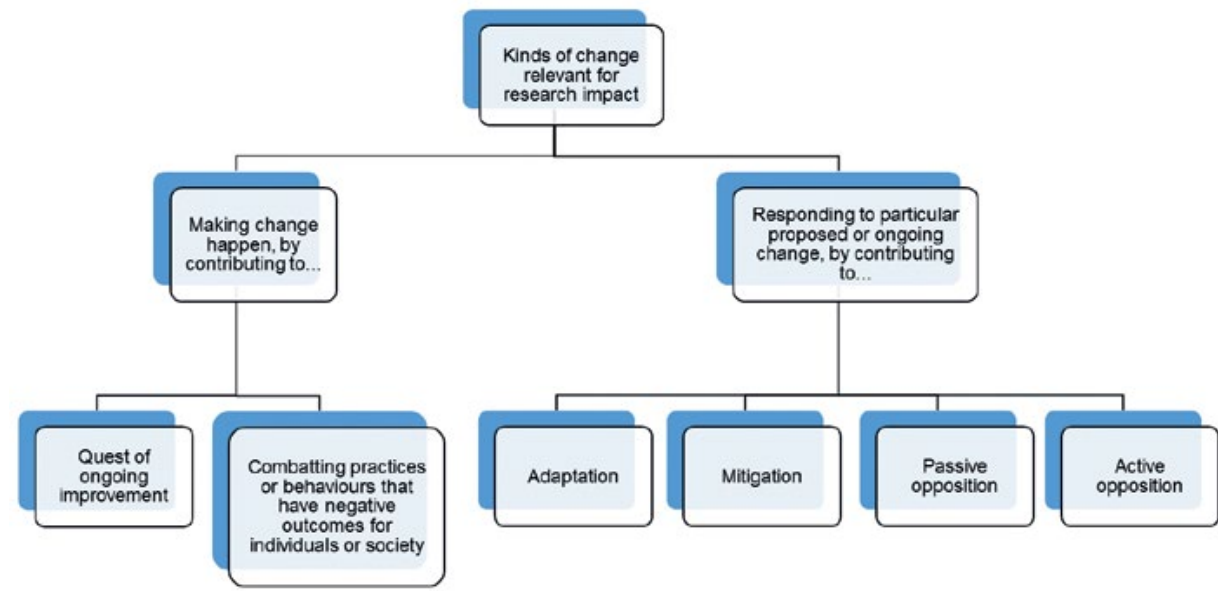

Figure 2 A framework for understanding and assessing kinds of change relevant for research impact.

Source: Author's illustration.

Two ways of making change happen that are important for research impact are 1) contributing to the ongoing quest for improvement and 2) combating practices or behaviours that have negative outcomes for individuals or society. Examples of contributing to the ongoing quest for improvement include technological research such as the development of wi-fi, and social research such as the development of an income-contingent loans scheme which aimed to provide accessibility to higher education in an environment where government 
provision of free higher education was no longer seen as sustainable. ${ }^{8}$ Tobacco control research is an example of combating practices or behaviours with negative outcomes for individuals and society.

Responding to change involves reacting to change that is proposed (such as the introduction of a new tax or the construction of a new dam) or that is already ongoing (such as global environmental change or the spread of an infectious disease). It can be useful to tease out four types of response along with how these are relevant to understanding research impact.

First, adaptation involves adjusting to the consequences of proposed or ongoing change, and is illustrated by demographic research on population ageing which highlights the need for altered housing, transport and infrastructure that is suited to the elderly (as suggested in Peter McDonald's chapter).

Second, mitigation involves influencing the path of proposed or ongoing change to lessen likely negative outcomes. This can be exemplified by a different kind of demographic research, such as examining the potential effects of increasing migration or measures to increase the birthrate as strategies for a country concerned about low population growth (as suggested in Peter McDonald's chapter). Adaptation tends to be less controversial than mitigation.

Third, passive opposition ${ }^{9}$ involves not complying with proposed or ongoing change. A hypothetical example would be research on how to avoid paying a new tax and its likely consequences for citizens and the government.

Finally, active opposition involves working to halt proposed or ongoing change. Examples include materials conservation research on ways to stop decay in museum artefacts (as suggested in Ian MacLeod's chapter) or anthropological research demonstrating the cultural value of a place scheduled to be destroyed by mining or other development (as suggested in Francesca Merlan's chapter).

Change can also be incremental, adaptive or transformational. Incremental change can be thought of as the tweaking of policies and practices over time in order, for example, to accommodate altered circumstances or respond to unintended consequences. Adaptive change or adaptive management, as described in Mark Stafford Smith's chapter, differs from incremental change in that it involves regular reassessment and retargeting in order to avoid triggering a change that cannot be recovered from. At the symposium Paul Griffiths (personal communication, 2013) provided a useful analogy likening incremental change to artillery fire, where each shot is followed by adjustments, depending on how close it got to the target.

8 See references to the work of John O'Sullivan and Bruce Chapman above.

9 Mitigation is differentiated from passive and active opposition here, but opposition could also be seen as a form of mitigation. 
By contrast, adaptive management is like a game of pinball, where the aim is not only to strike targets but also to avoid falling into the drain, which ends the game. Transformational change involves a radical reformulation of policy or practice. Each of the six types of change presented in Figure 2 could be incremental or transformational, whereas adaptive management applies to responses to ongoing change - such as global environmental change - where moving outside a safe operating space is a real possibility.

\section{Where the research is targeted}

The arena which is the target for change will influence the skills and techniques required, as well as the costs and risks to be negotiated. Further, any effective description and assessment of change is predicated on understanding the target(s). First, as shown in Figure 3, the primary target may be one or more of government, business or civil society. ${ }^{10}$ Second, the aim may be to affect policy, which can be usefully thought of as general guidelines and incentives influencing change, or it may be to affect practice, which is the actual implementation of measures to affect change. An example of research influencing government policy change is where demographic researchers advise governments to earmark funding to build more aged-care accommodation and help them figure out the proportions of different kinds of accommodation (e.g. independent living, assisted living and nursing care) to be funded. An example of research influencing civil society practice change is where public health researchers provide an online self-help program that citizens can use to tackle depression and anxiety.

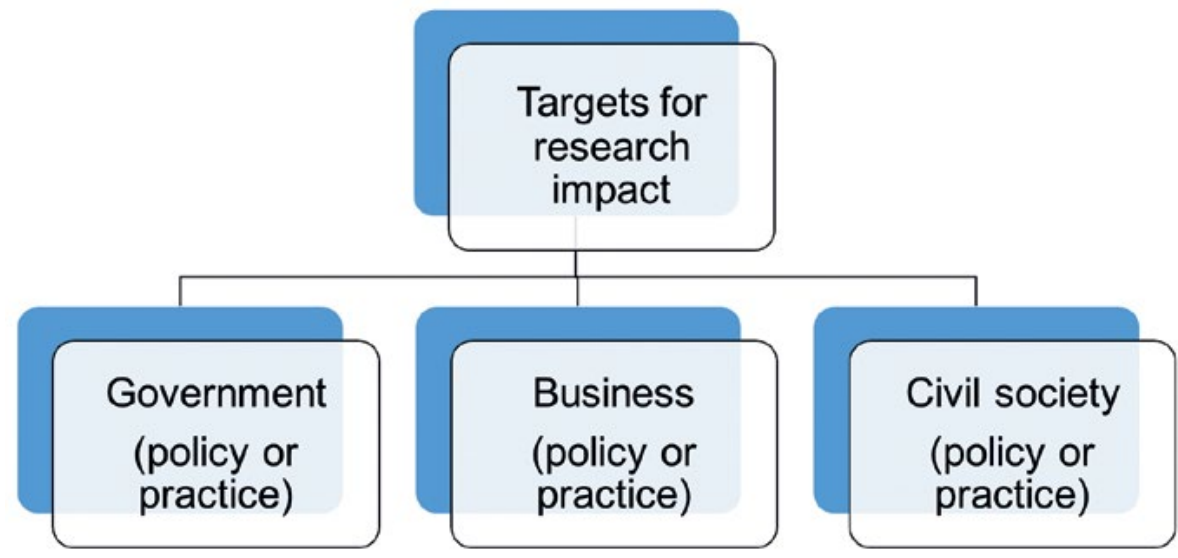

Figure $3 \mathrm{~A}$ framework for understanding and assessing targets for research impact.

Source: Author's illustration.

10 Civil society refers to non-government organisations including universities, as well as unorganised community groups such as residents of a particular area (such as a suburb affected by a toxic hazard) or sufferers of a specific disease (such as mesothelioma). 


\section{A new publication culture as the primary assessment structure}

If researchers are to be validated in taking an array of stances in relation to research impact, from relatively little involvement to being highly active, this must also be reflected in the reward system. Here I am not going to review the substantial literature on the requirement for new reward structures, or existing and proposed schemes. Instead the focus is on one way of assessing impact which does not seem to have been given much formal consideration, namely developing a peer-reviewed publication culture (Bammer 2014). I argue that having assessment structured around publications can potentially encompass both diverse kinds of researcher engagement with impact and also the complexities of the dynamic change environment.

There are three additional advantages of using peer-reviewed publication as the cornerstone of describing and assessing research impact:

- Publication is already the main accepted way for researchers to report on their findings, allowing the findings to be distributed, shared and built on.

- Peer-review is already the main accepted way for research findings to be assessed.

- In the longer term, peer-reviewed publication could allow for the development of effective metrics for assessing research impact.

Currently, coming to grips with how researchers can effectively negotiate the complexities of the dynamic change environment is hampered by lack of reporting and analysis. Much experience - both successful and unsuccessfulgoes undocumented and does not contribute to an overall fund of knowledge about achieving research impact. Instead, informal anecdotes are the main way researchers learn from each other about how to achieve and assess research impact. Only the most obvious successes are celebrated. A publication culture provides a platform for lessons to be shared.

In such publications, researchers engaged in driving change, for example, would describe what they set out to achieve, how they went about it, the context in which they operated along with the forces they encountered, what the outcomes were and, most importantly, the lessons learnt for the further development of theory and practice about attaining research impact. One consequence of the dynamic change environment is that what the researchers set out to achieve may be quite different from what they actually accomplished, so that highlighting both is an important aspect of publication. 
A critical outcome of a publication culture is to build a body of theory and practice about attaining research impact. The aim is not to identify foolproof formulae, but rather general principles and transferable processes that can be adapted to specific contexts. The aim is for such a body of theory and practice to encompass and build on not only the diversity of ways of achieving research impact described in the previous section, but also the complexities of the dynamic change environment.

As is the case now, journals and books would be the key publication formats, but it is also worth thinking about how a publication culture could embrace effective use of social media. Encouraging documentation and publication and assisting this by providing useful and agreed frameworks for writing about research impact will make more readily available a rich picture of what researchers undertake and how they handle the complexities of the dynamic change environment.

If we focus on journals, it is possible to imagine eclectic generalist journals and focused specialised ones. In fact examples of the latter already exist and are growing in numbers. They include Implementation Science and Science Translational Medicine, to mention two in the health domain. Established journals are increasingly including papers about implementation. It is also possible to imagine journals specialising in various aspects of research impact, with one journal focusing on research communication, another on driving change, and yet another on informing government policy change. Such journals would be particularly useful in sharing lessons across areas, for example, by enabling population health researchers to trade lessons with environmental and security researchers on effective problem-framing or engagement with social media.

A publication culture also provides scope for innovation in how implementation is reported. It is possible to imagine, for example, an impact on policy being analysed not only through the eyes of the researchers involved, but also through those of the policymakers they targeted. Even more ambitiously, all the involved parties (lobbyists, opponents, special interest groups, and so on) could be invited to contribute, providing a 360 degree view. Better than anything else this would illustrate the complexities and competing interests involved.

Peer-review would be the main assessment tool, because those who have realised or attempted to achieve impact themselves are in the best position to assess the efforts of others. Peer-review can accommodate existing discipline and area differences, because like will assess like. For example, economists have a privileged place in affecting government policy, as there is a tradition of economic research being called on by government policymakers. Economists would therefore assess each other, but not those from other disciplines whose history of influence is different. Similarly, some areas, such as tobacco control, 
have a longer history of engagement with policy and practice change than others, such as obesity reduction. The standards by which tobacco control efforts are assessed would therefore be different from those for assessing obesity reduction efforts, with different kinds of peers involved in the assessment. For peer-review to work, appropriate colleges of peers must be established.

Peer-reviewed publications can not only accommodate different forms and levels of research impact, but can also allow more complex attempts at research impact to be recognised and rewarded. For example, extra kudos may be given to those who achieve or seek to achieve impact on problems which require engagement with several targets, who tackle complex problems with no clear solutions, or who work with problems embedded in a highly volatile context.

The kind of metrics that work for assessing research findings through publication should also work for assessing research impact through publication. However, because the publication culture for research impact is still in its infancy, it will take some time for these metrics to become established and effective. Importantly, if metrics for both findings and impact count, it should be possible to accommodate the diversity of researcher enthusiasm for engaging with research impact, by allowing publications on findings to be traded off against publications on impact. This would allow some researchers to be assessed primarily by their publications on findings, while others are assessed primarily by their publications on impact; and there are, of course, a range of options in between. Researchers can therefore be judged against their choices in terms of engaging with research impact, in other words, how effective they are for the choices they have made, not by some arbitrary gold standard.

Finally, let us review the challenges of establishing a peer-reviewed publication culture for assessing research impact. First, keeping on top of the literature is already a major challenge for most researchers and this proposal will only add to that burden. It may, however, give added impetus to the work of information scientists in improving cataloguing and search strategies. Second, the peerreview system is far from perfect. But (to adapt Winston Churchill's assessment of democracy), I suggest that 'no one pretends that peer-review is perfect or all-wise. Indeed ... peer-review is the worst form of assessment except for all those other forms that have been tried from time to time ${ }^{\prime 1}$. Certainly there is work to be done in developing effective - and realistic — criteria for peer review of research impact.

\footnotetext{
11 In a speech to the House of Commons on 11 November 1947, Churchill described democracy thus: 'Many forms of Government have been tried, and will be tried in this world of sin and woe. No one pretends that democracy is perfect or all-wise. Indeed, it has been said that democracy is the worst form of Government except for all those other forms that have been tried from time to time.' From Churchill speaks: collected speeches in peace and war, 1897-1963, Chelsea House, London (1983), source www.goodreads.com/work/ quotes/1716637-churchill-speaks-1897-1963-collected-speeches-in-peace-war, accessed 28 February 2015.
} 
There are several other challenges relevant not just to establishing a peerreviewed publication culture, but for all ways of assessing research impact. Recognising and documenting them is a first step to developing effective ways of dealing with them.

The first is allocation of credit. As we have seen in some of the chapters and in the analysis of the dynamic change environment, researchers play one role among many. In a change to government policy, for example, critical roles may also be played by the relevant minister and other politicians; various public servants; specific reporters, editors and owners in the media; and particular members of lobby groups. Teasing out the relative value of each of these contributions is impossible because their effects are neither additive nor independent.

A second challenge is that at least some change is most effective when no public credit is taken. Ian MacLeod (personal communication, 2013) provided an example in the symposium discussion [identifiers have been removed]:

My work on [Indigenous] rock art showed there was massive acidification. I was concerned about it. I got a briefing with the minister. When the minister heard about it she was alarmed and she said, 'Well, are you going to leak it to the media?' and I said, 'No minister, of course not,' and she said, 'I'll organise a briefing with [company]'. So I briefed [company], they didn't like what I said. They analysed the data and then they spent four and a half million dollars rapidly introducing different types of burners, and they reduced their $\mathrm{NO}_{\mathrm{x}}{ }^{12}$ emissions by a factor of six times and then nothing was said. It didn't come out in the media, only you around here know it and the ministers involved, but see there are other ways of bringing about change, because all I was concerned was with the fate of the rock art. I didn't mind how I got the change done, but the change was managed that way and so it was hair-raising but good.

A third challenge is assessing partial success and failure. How should we evaluate a good process that produced no outcomes, for example because political circumstances changed? What about research that produced successful change but also led to major unintended adverse consequences? What credit should be given to impact primarily produced by luck rather than a good process or good research findings? What about the successful uptake of research findings that result from poorly designed studies or that have been incorrectly interpreted? A way through such dilemmas needs to be found, so that rewards are appropriate and researchers are not dissuaded from attempting to achieve research impact.

$12 \mathrm{NO}_{\mathrm{x}}$ is a generic term for the mono-nitrogen oxides $\mathrm{NO}$ (nitric oxide) and $\mathrm{NO}_{2}$ (nitrogen dioxide). 
In this section I have laid out a specific proposal that would allow research impact to be more effectively described and assessed. It builds on the dominant peer-reviewed publication culture for describing, distributing and evaluating research findings, as well as existing trends evidenced by the growth of journals specialised on impact in particular areas. There are major challenges. No system to measure research impact will be problem-free and this applies to existing and mooted schemes. A peer-reviewed publication culture will also not provide a perfect solution, but it may be good enough.

\section{Conclusion}

This chapter seeks to provide fresh thinking about research impact by approaching it through a new consideration, namely that research impact involves a process of negotiating a dynamic change environment. The multidisciplinary author perspectives in this book's chapters and the symposium discussion illustrate the complexities encompassed by change. In turn, the complexities underpin three topics that have been largely neglected to date, each of which is also illustrated by the chapters and discussion. For achievement and assessment of research impact to become core research activities, effective ways must be found to address the associated opportunity and transaction costs, as well as potential challenges to research integrity.

It must be possible for researchers to choose greater or lesser involvement with achieving research impact. Reward systems need to be flexible enough to accommodate and validate this range of choices. An essential requirement is effective ways to describe different kinds of research impact and a start is made here by proposing frameworks for three primary ways that research can have impact, for different kinds of change and for different targets. A proposal is also made for how a peer-reviewed publication culture about research impact could provide an effective basis for assessment.

To round this chapter off, it is worth asking some big questions. There is no doubt that change should be informed by research evidence. But how much should the whole research enterprise move beyond discovery to implementation? Political engagement which is fundamental to much of the achievement of research impact is at odds with the rational detachment required of researchers (see also Gadlin and Bennett, 2013). If researchers are not to be the main players in achieving research impact, then who? If (some) researchers are to be the main players, how can they best be supported and rewarded? 


\section{References}

Bammer, G (2013) Disciplining interdisciplinarity: integration and implementation sciences for researching complex real-world problems. ANU E Press, Canberra. http://press.anu.edu.au?p=222171.

Bammer, G (2014) Studying the effects of studies. Australian Financial Review (Education Section), 17 February: 35.

Gadlin, H and Bennett, LM (2013) Interdisciplinarity without borders. Commentary in Bammer, G Disciplining interdisciplinarity: integration and implementation sciences for researching complex real-world problems. ANU E Press, Canberra. http://press.anu.edu.au?p=222171.

Marx, K (1977, originally published in 1852) The eighteenth Brumaire of Louis Napoleon. In D McLellan, ed. Karl Marx: selected writings. Oxford University Press, Oxford: 300-25.

\section{Appendix: Multidisciplinary research and Integration and Implementation Sciences}

This chapter has provided an example of how multidisciplinary research can be synthesised. In this appendix I review the strengths and weaknesses of multidisciplinary research and provide a brief overview of Integration and Implementation Sciences (I2S), which has underpinned my approach to organising this project.

\section{Multidisciplinary research and its synthesis}

As described in Chapter 1, the approach used in this book is multidisciplinary. Every contributor tackled the issue of change as they saw fit. There was no attempt to start with a shared framework or a common specific aspect of change. A multidisciplinary approach therefore allows the richness of different perspectives to be demonstrated.

Another strength of a multidisciplinary approach is that these perspectives can be combined in many different ways. Anyone can tailor-make their own synthesis by selecting the points that are meaningful and useful to deepen their understanding of change-related issues that concern them. In this chapter I have tailor-made a synthesis around my interest in research impact. In Chapter 1 I also flagged a longer term interest in using the perspectives in this book to start a process for providing general principles to guide thinking about change, 
and a roadmap to the specific approaches to change of different disciplines and practice areas. Others could use the same material for different syntheses, such as developing a toolbox of novel strategies for responding to escalating health care costs or examining similarities and differences in leading change at international, national and local levels.

A multidisciplinary approach is most useful in two situations. One is in dealing with projects that are broad and relatively unconstrained, rather than those which have a specific focus. Two of the earlier examples are relevant: developing general principles to guide thinking about change and a roadmap to specific disciplinary and practice interests, as well as examining change at international, national and local levels. The second is when attempting to move thinking on a topic away from conventional lines, and to generate fresh approaches. This is the purpose in this chapter on research impact and would, for example, also be the case when developing a toolbox of novel strategies for responding to escalating health care costs.

There are diverse ways in which synthesis can be undertaken. As mentioned in the examples above, individuals who are readers of the contributed perspectives can do the synthesis, or it can be a group process. Synthesis can also be undertaken by the contributors themselves: the whole group, a subgroup, or an individual contributor. As in this project, the synthesis can also be undertaken by the organiser, with the written contributions embellished by a gathering of the authors in a symposium. Individual synthesisers will generally build on pre-existing expertise or interest. Group synthesisers will usually add to this by using dialogue to draw together and build on their members' expertise and reflections on the contributed perspectives; this can involve formal dialogue methods, informal processes, or a combination of these.

The strengths of a multidisciplinary approach also contain the seeds of its weaknesses. The main challenge with multidisciplinary research comes in synthesising the contributions, as it is not certain what the points of intersection will be with the interests of the person undertaking the synthesis, or even if there will be any. Synthesis requires considerable, time-consuming sifting of the material. Care must also be taken in interpretation, especially when points of interest to the synthesiser are relevant in a different context from that in which they were presented by the contributor.

Other challenges in multidisciplinary research are common to all research involving multiple perspectives. In particular, the value of the disciplinary and practice perspectives is greatly dependent on the expertise of the contributors. And the choice of contributors relies on the skills of the project leader or organiser. 
To round off this discussion, let us briefly compare multidisciplinary research with interdisciplinary and transdisciplinary research (I will not differentiate between the latter two here). In interdisciplinary and transdisciplinary research, the project commences by establishing a shared focus, and often also a common framework, for working together. This necessarily and deliberately limits which perspectives are brought to bear and the approach they take. Rather than the wide-ranging treatments of a topic found in multidisciplinary research, the disciplinary and practice perspectives in interdisciplinary and transdisciplinary research are directed to the agreed shared focus of the overall project. Synthesis of these perspectives is then much more straightforward, because the focus for it is established when the project commences. Each approach-multidisciplinary, interdisciplinary and transdisciplinary - therefore has advantages and disadvantages. It is important to choose the approach most suitable for the project at hand.

This appendix concludes with a brief introduction to I2S, which has motivated my interest in change. I2S supports multidisciplinary, interdisciplinary and transdisciplinary research.

\section{Introducing Integration and Implementation Sciences}

My role in the project is as a specialist in the emerging discipline of I2S. The primary focus of I2S is to improve research contributions to tackling complex problems like global environmental change, organised crime and escalating health care costs. To this end, I2S is developing a repository of methods and concepts, along with illustrative case examples, that researchers can draw on.

A core component of I2S is to provide (and continue to develop) tools that researchers can use to contribute to policy and practice improvements in how complex problems are responded to. These include understanding how relevant policy and practice arenas operate and where research inputs fit, as well as practical ways of communicating and engaging with policymakers and practitioners.

I2S also recognises that the strength of the evidence is critical. For complex real world problems this requires synthesis of knowledge from all the relevant disciplines and practice areas. I2S tools include methods for determining which perspectives are most useful, as well as for synthesising them. But implementing research based only on the best available evidence is not enough; due consideration must also be given to what is not known. Ignoring unknowns can lead to unpleasant surprises and unintended adverse consequences, and I2S provides tools for mitigating these problems. 
Providing research support for policy and practice change, synthesising knowledge, and understanding and managing diverse unknowns are the three domains of I2S. Each domain is structured around a five question framework which provides a systematic approach to organising the repository of methods, concepts and case studies. A detailed description of I2S can be found in Bammer (2013).

The novel aspects of I2S are:

1. the three domains and five-question framework, which aim to provide an effective and efficient way of gathering and organising widely dispersed concepts and methods relevant to tackling complex real-world problems

2. thinking of integration as bringing together both what is known and what is not known about the problem of interest (rather than only synthesising knowledge) to support policy and practice change

3. taking on the job which is currently no one's business, that of drawing together knowledge about change, so that it is better understood and managed, particularly by researchers contributing to tackling complex problems

4. arguing for a new discipline and for including this disciplinary expertise in teams seeking to have research impact on complex real-world problems.

I2S therefore aims to contribute to strengthening research impact in multiple ways: by providing a one-stop shop for methods and concepts for achieving research impact, as well as illustrative case studies; by providing ways to deal with unknowns, especially as triggers to unintended consequences; and by highlighting the importance of understanding change and, in the longer term, providing general principles to guide thinking about change, and a roadmap to the specific issues covered by different disciplines and practice areas. 


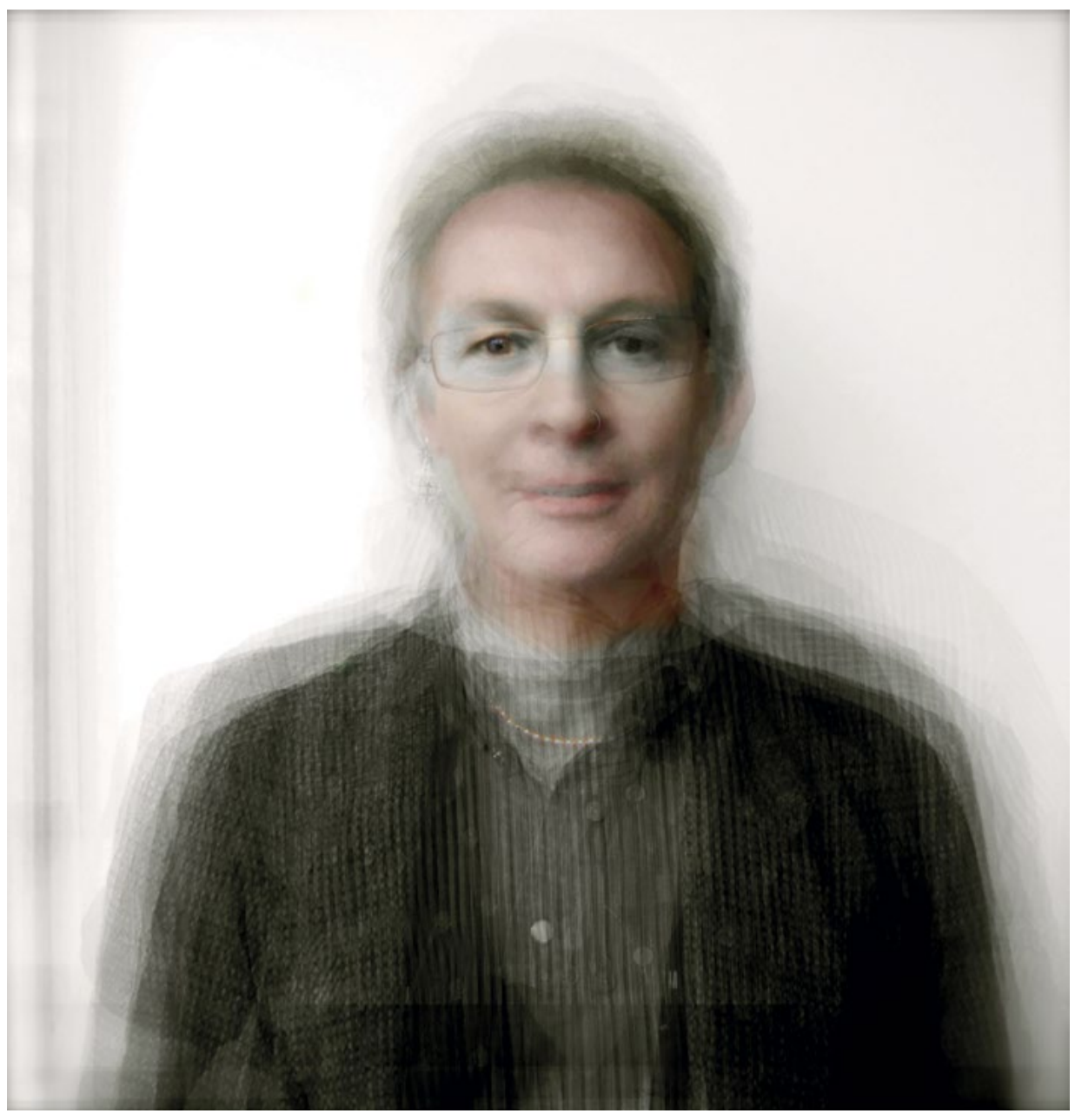

Integration of the change symposium participants.

Source: Frank Thirion, 2013, amalgamating individual photographs taken by John Reid, 2013. 
This text is taken from Change! Combining Analytic Approaches with Street Wisdom, edited by Gabriele Bammer, published 2015 by ANU Press, The Australian National University, Canberra, Australia. 